\title{
Optimization of Train Speed in Heavy Density of Indian Railway Traffic using Genetic Algorithm
}

\author{
Soumya Sengupta ${ }^{1}$, Dr. Amartya Neogi ${ }^{2}$ \\ ${ }^{1}$ Department of Computer Science and Applications, Panskura Banamali College, Vidyasagar University, India \\ ${ }^{2}$ Department of Computer Science and Engineering, Dr B.C. Roy Engineering College, West Bengal University of Technology, India
}

\begin{abstract}
The railway transportation system has much advantage in eco-friendliness, punctuality and safety compared to any other transportation system. Most of the railway system administrators have to control and operate under limited resource such as trains and facilities. It is necessary to control travelling time for efficient operation in the railway system because the board rate of passenger is inconstant with time variance. In this study, a new optimization method considering operation time is proposed by using genetic algorithm. The intelligent train speed regulation algorithm plays a very important role in the automatic train control system. This algorithm can be used to improve passenger travel comfort at each defined inter-section of travel time. In this algorithm each travel time target speed will be reflected by its particular speed profile, which has been optimized by genetic algorithm.
\end{abstract}

Keyword: Genetic Algorithm, Indian Railway, Passenger Demand, Railway Speed, Timetable

\section{Introduction}

Indian Railway one of the biggest railway network around the world. To operate efficiently and effectively train must operate according to published timetable. To maintain predefined timetable train must need to maintain certain speed. It is very difficult and challenging to maintain predefined speed in heavy density of railway traffic area. Although to identify heavy density railway traffic area is depends on some parameter such as train timing, no. of trains, passenger demand, no. of tracks and platforms etc. It also depends on some uncontrolled variable which may changes in accordance with nature of parameter.

Genetic Algorithm is a heuristic research method that imitates the process of natural evolution. It is motivated by the principles of natural selection and survival of the fittest individuals. This method in commonly used to generate useful solutions to optimization problems. To optimize the said problem especially for busy passenger railway system is naturally broken down into several stages. After getting the existing proper input data using genetic algorithm methodology find out the alternatives to achieve the desire goal of accuracy of existing timetable.

\section{Literature Review}

Several authors were developed different techniques and approaches to address for optimizing railway speed to keep timetable periodic. Some of these are-

[1] kroon et al. (2007) proposed the generation of the departure and arrival times and the selection of the routes through the stations. The author define a set of different types of constraints and them as an input to the optimization model namely PESP solver and obtaining a timetable that will be use to another input of routing model. Finally, it presents an optimization model for maintaining certain train speed for follow existing timetable.

[2] Thomas et al. (2008) focus on single train based train speed optimization that includes full acceleration, cruising, coasting and full breaking for a particular region specially urban railway area, where the cruising stage is unnecessary unless the maximum speed is reached after full acceleration.

[3] An approach by London underground through until 2008 , which was the subject of a paper presented by Wallace et al. (1995) through metro operation have rather simpler timetabling challenges than mainline railways.

[4] Zwanereld et al. (1996) consider the problem for maintain train speed through heavy traffic railway region, given the layout of the station and a proposed predefined timetable they determine whether there is a feasible routing for the trains with it operation at maximum speed with satisfying safety rules and train service or connection constraints.

[5] River et al. (1996) developed methods for finding good or acceptable solutions for this combinational problem. They emulate some of these methods of one train at a time, dealing with trains chronological order sub-divisions into speed classes etc. for input and output manipulation and display the information particularly applicable for a heavy density of railway traffic area.

[6] J. Munoz-Paniagua, J. García et al. (2011) from their concept an aerodynamic optimization of the train aerodynamic characteristics in term of front wind action sensitivity is carried out. A genetic algorithm (GA) is used to perform a shape optimization study of a high-speed train nose. They are trying to shows the feasibility of using GA in combination with Artificial Neural Network for this problem. They used meta models for this purpose. The basic idea of metamodels is to construct approximations of the analysis codes or numerical solvers that are more e-cient to run, enabling a faster evaluation and optimization process. Using a genetic algorithm as an alternative optimization tool has been tested and good results have been obtained.

[7] RuidanSu, Qianrong Gu et al. (2014 ) proposed A parallel multi population genetic algorithm (PMPGA) is proposed to optimize the train control strategy, which 


\section{International Journal of Science and Research (IJSR) \\ ISSN (Online): 2319-7064 \\ Index Copernicus Value (2013): 6.14 | Impact Factor (2015): 6.391}

reduces the energy consumption at a specified running time. They also proposed not only energy consumption, but also running time, security, and riding comfort. They are trying to compare the PMPGA with the multi objective fuzzy optimization algorithm and differential evolution based algorithm and showed that PMPGA has achieved better result. A SGA and PMPGA were applied to find a perfect running based on a specified time.

[8] Haichuan Tang, C. Tyler Dick et al. from their concept Traditional Automatic Train Operation (ATO) algorithms are generally designed based on single-train operation with the objective of improving the speed profile of a single train to reduce mechanical energy consumed under operational constraints. Genetic algorithms have been applied to search for the optimal train speed profiles. Tractive/braking efforts of both trains and energy cost at substation level are defined as strings of chromosome and the fitness function respectively. To demonstrate visual $\mathrm{C}++$ platform demonstrates that the algorithm can provide optimal train speed profiles with better energy performance while satisfying operational constraints. A mathematical energy consumption model of bidirectional trains. Genetic algorithms have been applied to generate an optimal speed profile for the second train to minimize energy consumption at the power substations. Running in the same power section has been established based on train operations and electrical theories.

[9] Youneng Huang, Tao Tang et al. (2015) proposes an energy-efficient approach to reduce the traction energy by optimizing the train operation for multiple inter-stations. Both the trip time and driving strategy are considered in the proposed optimization approach. Firstly, a bi-level programming model of multiple inter-stations is developed for the energy-efficient train operation problem, which is then converted into an integrated model to calculate the driving strategy for multiple inter-stations. Additionally, the multi-population genetic algorithm (MPGA) is used to solve the problem, followed by calculating the energy-efficient trip times. Based on the operation data from the Beijing Changping subway line, a numerical example is given to prove that the proposed method can reduce the energy consumption of multiple inter-stations by $6.16 \%$.

[10] Hiraoki kitano et al. (1990) reported results of systematic experiments designed to compare the speed of convergence for training neural networks using genetic algorithms and gradient descent methods. At the outset of this paper, they pointed out that simple application of genetic algorithms would be outperformed by back propagation due to its weak local fine-tuning capability and computational cost for evaluating numbers of chromosomes in one generation. Thus, instead of directly comparing genetic algorithms and back propagation, they have compared the GA-BP method and quickprop, both of which are faster methods of training neural networks. The neural network training task was confirmed to have less compositional feature combinations of good partial solutions do not necessarily create a better overall solution. Thus, the speed of convergence of genetic algorithm-based training was significantly undermined.
[11] Xin Feng ,Jinbao Luo et al. (2013) the factors with passenger's travel demand , passenger's need of comfort and the wasted train capacity. On the basis of the factors, they provides an wide at solve high-speed passengers train operation plan problem. First, all the possible train operation plans are listed, and then with the aid of intelligent algorithm those train operation plans which cannot be send edout are excluded. Finally, there st of the train operation plans just can meet passenger's travel demand. The paper uses the genetic algorithm to get the best solution and will get train operation quickly.

\section{Problem Analysis}

Many optimization approaches has been going on since many year. In the other countries the train speed is faster than our countries and comfort zone for the passenger is almost good and they almost maintain the train time-table .For this purpose many modeling and frame work was done.

But in Indian railway it is too difficult to maintain train speed in heavy density area. There are many reasons for this purpose like railway infrastructure, no of platforms, natural disasters, too many cross over, passenger issues, signal problem etc. Now a day for our country the decision of some of thing is taken manually in different region. But if we optimize this problem using genetic algorithm then it is on behalf of passenger facility. To maintain the train time table we have to increase all the train speed. Even if in heavy density area the train speed will remain same. It is a challenge to maintain the speed of train in Indian railway service for heavy density area without involving the security rule. So we are trying to optimize the train speed using genetic algorithm and also maintain the railway train time table.

\section{Genetic Algorithm}

Genetic Algorithm is search algorithm based on the mechanism of natural selection and natural genetics. They combine survival of the fittest among string structure with a structured yet randomized information exchange to form a search algorithm with some of the innovative flair of human search. In every generation a new set of artificial creatures is created using bits and pieces of the fittest of the old; an occasional new part tried for good measure. In this paper we used Genetic Algorithm in following manner to optimize desire output.

Step I [Start] Generate random population of chromosomes, that is, suitable solutions for the problem.

Step II [Fitness] Evaluate the fitness of each chromosome in the population.

Step III [New population] Create a new population by repeat following steps until the new population is complete.

a) [Selection] Select two parent chromosomes from a population according their fitness. Better the fitness, the bigger chance to be selected the parent.

b) [Crossover] With a crossover probability, cross over the parents to form new offspring, that is, children. If no crossover was performed, offspring is the exact copy of parents. 


\section{International Journal of Science and Research (IJSR) \\ ISSN (Online): 2319-7064 \\ Index Copernicus Value (2013): 6.14 | Impact Factor (2015): 6.391}

c) [Mutation] With a mutation probability, mutate new offspring at each locus.

d) [Accepting] Place new offspring in the new population.

Step IV [Replace] Use new generated population for a further run of the algorithm.

Step V [Test]If the end condition is satisfied, stop, and return the best solution in current population.

Step VI [Loop] Go to step 2.

\section{Planning and Analysis of Data}

To optimize our desired goal we segmented our proposed job into some sub-categories which are associated with genetic algorithm existing methodology. First of all, we have to collect or gathered existing information related to our problem (such as no. of tracks, passenger demand, traffic density etc.). Next, we match with genetic algorithm parameter and build gene, chromosome and genotype. Based on the priority and existing database we select the fitness ratio using selection procedure and build some parent for crossover to find out the new combination of chromosome. Out of many procedures we choose some popular crossover technique like one-point crossover, multi-point crossover and uniform crossover etc. After successful operation we will get a new setup chromosome out of many available list, then we choose the best possible combination and muted it for breeding. Then new generation of combination test the optimality of our existing approach if they not satisfied then we reconstruct a new setup chromosome and do the same operation for new combination of breeding. If the new generation satisfies our optimality approach then we set the new combination of genotype result outcome. Then we find our optimal approach after 2 or 3 breeding process then we regenerate a new sample and repeat same procedure until desire goal have been

\section{Test of Optimality}

Most of the railway administrator has to control and operate train speed under many resources in which some parameters are controllable and some are uncontrollable. Among the controllable parameters there are many aspects to increase the speed. We mainly focus the following three parameters for our proposed work........

- Passengers demand

- Railway traffic density

- Number of track

We have to keep in mind the passenger demands in time to time. We have to ensure the passengers can reach the destination in right time. For this we strictly maintain the predefined time table and to maintain timetable we have to optimize the train speed. It is very difficult to maintain train speed in heavy density area. In heavy density area we have find out numbers of tracks, number of platforms, and number of railway crossing etc. at the particular time. In such cases we have to reduce the worst time by the train and for this we have to increase train speed. Number of tracks a essential parameters for increasing train speed. At first we have to figure out number of track is available and not occupied by other trains. Also we have to check the distance between two trains in same track and there speed. According to the above condition we have to optimize the train speed. We always try to find the best possible combination of our research problem. Our basic three optimality parameters are passenger demand, no. of track and railway traffic density. Although there are so many parameters which take very important role for the said research problem. But we only consider above three parameters to find out the optimal solution. Among three constraints if we consider passenger demand we must try to keep it as high as possible. But there is a limitation for no. of track. In case if railway traffic density it may be high or low. But the problem is that, if the railway traffic density is maximum then passenger demand will not be fulfilled which is not our destiny. That is why, we try to some intermediate value which may act as optimal value.

To find out our best possible optimal solution we consider individual set of genes of each constraints and each of which four individual genes. Finally, we construct a chromosome which contains 6 pair of genes that may combination of 000000000000 to 111111111111 . If we extract this chromosome with each four genes that ranges illustrate below-

Table 1: Optimality Table

\begin{tabular}{|c|c|c|}
\hline Passenger demand & Railway traffic density & No. of track \\
\hline 0000 & 0000 & 0000 \\
\hline 0001 & 0001 & 0001 \\
\hline 0010 & 0010 & 0001 \\
\hline 0010 & 0010 & 0010 \\
\hline 0011 & 0011 & 0011 \\
\hline 0100 & 0100 & 0100 \\
\hline 0101 & 0101 & 0101 \\
\hline 0110 & 0110 & 0110 \\
\hline 0111 & 0111 & 0111 \\
\hline 1000 & 1000 & 1000 \\
\hline 1001 & 1001 & 1001 \\
\hline 1010 & 1010 & 1010 \\
\hline 1011 & 1011 & 1011 \\
\hline 1100 & 1100 & 1100 \\
\hline 1101 & 1101 & 1101 \\
\hline 1110 & 1110 & 1110 \\
\hline 1111 & 1111 & 1111 \\
\hline
\end{tabular}

Here, the minimum value is- 0000, maximum value is- 1111 and others are the intermediate value for optimization for each parameters. For example, if we try to maximize passenger demand and railway traffic density with a arbitrary genotype value 1010 and 1000 then we get some achievable solution. But, keeping without changing of no. of track which may denote by the value of 0101 . Now, we try to satisfy our optimality by provisionally increasing of passenger demand as well as traffic density. Only when the value of traffic density and passenger demand are reached tense to saturated value then only third variable (no. of track) might be changed slightly. We test the all genotype value using MATLAB simulation getting some set of optimality value out of them particularly a range of value may be accepted and other vary far of our achievable destiny. The simulated value and optimal range can be shown in following table from the result of MATLAB. 


\section{International Journal of Science and Research (IJSR) ISSN (Online): 2319-7064}

Index Copernicus Value (2013): 6.14 | Impact Factor (2015): 6.391

Table 2: Optimality table with lower cut off value

\begin{tabular}{|c|c|c|}
\hline Passenger demand & Railway traffic density & No. of track \\
\hline 0000 & 0000 & 0000 \\
\hline 0010 & 0100 & 0011 \\
\hline 0100 & 0101 & 0011 \\
\hline 0101 & 0101 & 0011 \\
\hline 0110 & 0110 & 0011 \\
\hline 0111 & 0110 & 0100 \\
\hline 1000 & 0110 & 0100 \\
\hline
\end{tabular}

Table 3: Optimality table with accepted value

\begin{tabular}{|c|c|c|}
\hline Passenger demand & Railway traffic density & No. of track \\
\hline 1010 & 0111 & 0101 \\
\hline 1011 & 1000 & 0101 \\
\hline
\end{tabular}

Table 4: Optimality table with upper cut off value

\begin{tabular}{|c|c|c|}
\hline Passenger demand & Railway traffic density & No. of track \\
\hline 1100 & 1001 & 0101 \\
\hline 1101 & 1001 & 0101 \\
\hline 1110 & 1010 & 0110 \\
\hline 1111 & 1011 & 0110 \\
\hline
\end{tabular}

From the MATLAB simulation of considered combinational value of genotype we achieved some optimal combination which shown in table 1 . From the above table we can say that, if no. of track is less then passenger demand is not fulfilled. In the other hand, if passenger demand and traffic density both high then we need to increase no. of track, but it has a limitation. So, we are trying to find a range of intermediate value which is optimal. In this approach the optimal range is 101001110101 to 101110000101 . Now we apply our proposed approach of crossover and then find a new combination of chromosome. If the new combination is almost same to our optimal value then we can say that, the desire solution is achieved. Otherwise, we have to take a new set of chromosome and reconstruct the crossover until the desire solution achieved.

\section{Methodology}

This paper considered Optimization of train speed in heavy density area of Indian railway traffic using genetic algorithm as a choice for and accelerated transit railway operation scheme for optimizing railway speed depends on different natural parameters.

Table 5: Comparison Table

\begin{tabular}{|c|c|c|}
\hline Real Life & Genetic Algorithm & Railway Module \\
\hline String & Chromosome & Timetable \\
\hline Character & Gene & Railway Operational Speed \\
\hline Population & Genotype & Passenger Demand \\
\hline $\begin{array}{c}\text { Generation } \\
\text { Procedure }\end{array}$ & Breeding & $\begin{array}{c}\text { Finding Optimal Speed For } \\
\text { maintaining new schedule }\end{array}$ \\
\hline
\end{tabular}

Now we try to find out the best possible combination for above three constraints which satisfy our said optimality. To do this we will use the following work flow as bellow.

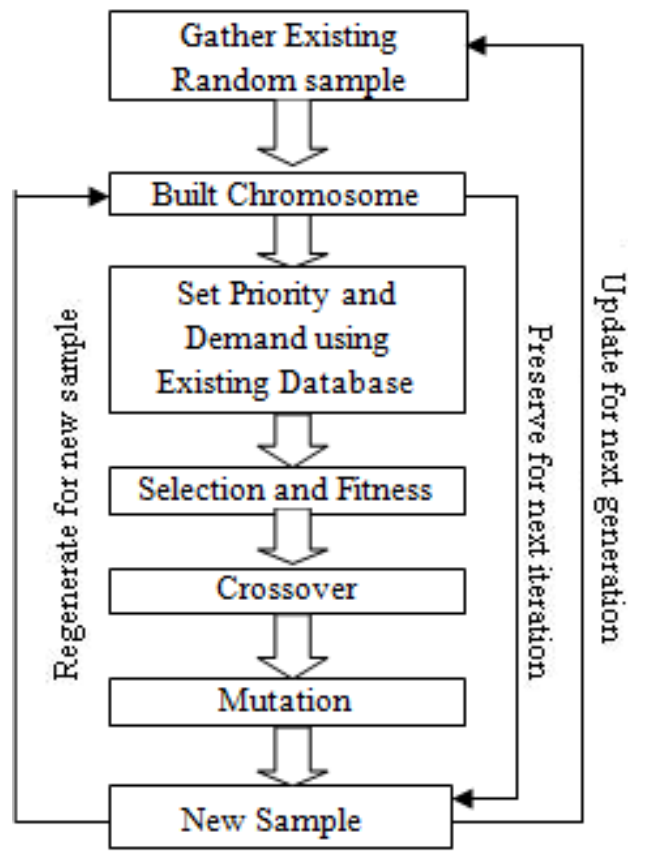

Figure 1: Proposed Genetic Algorithm to search for improves optimality of railway speed.

For example we choose two chromosome using fitness selection techniques, assume two chromosomes are 011101100100 and 110010010101 . Now we try to crossover of this chromosome and breed a new pair of child chromosome.

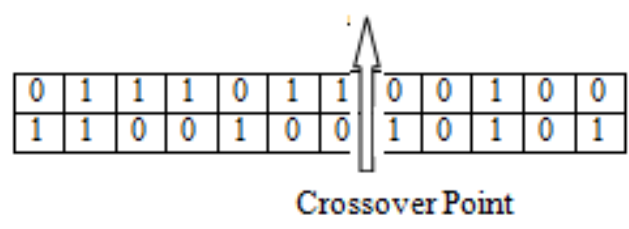

Figure 2: One point Crossover

The new schedules created after successful completion of crossover phase and are then passes through the mutation module. If the new schedule is satisfy our optimality range then we conclude a new schedule of railway speed for optimization. If not then we have rebuild a new pair of chromosome for breeding. In our example the resultant child chromosomes are not satisfy our predefined optimality range. We will again continuing this process until desire condition has been satisfied.

\section{Conclusion}

However there are several practical and theoretical problem to be solved but some influence cannot be controlled. By using our proposed study we will optimize time delay but sometime may not get fruitful result due to some inconsiderable constraints and their activity. In this research it does not address riders' behavior that is an individual passenger travelling from an origin to destination. In this paper we try to optimize rail speed with respect to three challenging constraints for some selected Indian railway divisions. Our overall focus is that to minimize passenger travel time and satisfying increasing passenger demands but do not hampering Indian railway security rules. 


\section{International Journal of Science and Research (IJSR) \\ ISSN (Online): 2319-7064}

Index Copernicus Value (2013): 6.14 | Impact Factor (2015): 6.391

\section{Acknowledgement}

First of all we would like to thankful of Indian Railway for their necessary information provided us. Without their support we cannot perform this challenge. We would also like to thank all staff of CS department of Panskura Banamali College. The authors also acknowledge to Mrs Arpita Sengupta for her massive assist of said research work.

\section{References}

[1] Fay, A., 2000. A fuzzy knowledge-based s ystem for railway traffic control. Engineering Applications of Artificial Intelligence 13 (6), 719-729.

[2] Kroon, J. M., Bakker, N. J., Smit, H. J. P., Liska, P., Thampi, K. R., Wang, P., ... \& Würfel, U. (2007). Nanocrystalline dye-sensitized solar cells having maximum performance. Progress in Photovoltaics: Research and Applications, 15(1), 1-18.

[3] Lever, J., Wallace, M., \& Richards, B. (1995). Constraint logic programming for scheduling and planning. BT technology journal, 13(1), 73-80.

[4] Delorme, X., Gandibleux, X., \& Rodriguez, J. (2009). Stability evaluation of a railway timetable at station level. European Journal of Operational Research, 195(3), 780-790.

[5] Huisman, D., Kroon, L. G., Lentink, R. M., \& Vromans, M. J. (2005). Operations research in passenger railway transportation. Statistica Neerlandica, 59(4), 467-497.

[6] Weihua, Y. M. Z. J. Z. (2012). Running Safety of Highspeed Trains on Bridges under Strong Crosswinds. Journal of Mechanical Engineering, 18, 018.

[7] Muñoz-Paniagua, J., García, J., \& Crespo, A. (2014). Genetically aerodynamic optimization of the nose shape of a high-speed train entering a tunnel. Journal of Wind Engineering and Industrial Aerodynamics, 130, 48-61

[8] Tang, H., Wang, Q., \& Dick, C. T. (2014, April). Optimizing Train Speed Profiles to Improve Regeneration Efficiency of Transit Operations. In 2014 Joint Rail Conference (pp. V001T07A004V001T07A004). American Society of Mechanical Engineers.

[9] Tang, H., Dick, C. T., \& Feng, X. (2015). Improving Regenerative Energy Receptivity in Metro Transit Systems: Coordinated Train Control Algorithm. Transportation Research Record: Journal of the Transportation Research Board, (2534), 48-56.

[10] Huang, Y., Ma, X., Su, S., \& Tang, T. (2015). Optimization of Train Operation in Multiple Interstations with Multi-Population Genetic Algorithm. Energies, 8(12), 14311-14329.

[11] Hwang, H. S. (1998). Control strategy for optimal compromise between trip time and energy consumption in a high-speed railway. Systems, Man and Cybernetics, Part A: Systems and Humans, IEEE Transactions on, 28(6), 791-802.

\section{Author Profile}

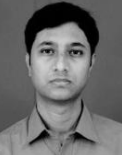

Soumya Sengupta is Faculty of Dept. of Computer Science and Applications, Panskura Banamali College, Vidyasagar University, India

Dr Amartya Neogi is Faculty of Computer Science and Engineering, Dr B. C. Roy Engineering College, West Bengal University of Technology, India. 